\title{
Liver Complete Response GvHD
}

National Cancer Institute

\section{Source}

National Cancer Institute. Liver Complete Response GvHD. NCI Thesaurus. Code C126706.

Normal ALT, alkaline phosphatase, and total bilirubin after previous elevation of 1 or more following GvHD involvement. 